\title{
$\angle S$ Research Square \\ Ketogenic diets induced glucose intolerance and lipid accumulation in mice with alterations in gut microbiota and metabolites
}

Yue Li

SJTU: Shanghai Jiao Tong University

Xin Yang

SJTU: Shanghai Jiao Tong University

Jing Zhang

SJTU: Shanghai Jiao Tong University

Tianyi Jiang

SJTU: Shanghai Jiao Tong University

Ziyi Zhang

SJTU: Shanghai Jiao Tong University

\section{Zhiyi Wang}

SJTU: Shanghai Jiao Tong University

\section{Mengxue Gong}

SJTU: Shanghai Jiao Tong University

\section{Liping Zhao}

SJTU: Shanghai Jiao Tong University

Chenhong Zhang ( $\square$ zhangchenhong@sjtu.edu.cn )

Shanghai Jiao Tong University https://orcid.org/0000-0002-4462-892X

\section{Research}

Keywords: Ketogenic diet, Gut microbiota, Fecal metabolites, Glucose and lipid metabolism

Posted Date: November 17th, 2020

DOl: https://doi.org/10.21203/rs.3.rs-108395/v1

License: (c) (i) This work is licensed under a Creative Commons Attribution 4.0 International License.

Read Full License

Version of Record: A version of this preprint was published at mBio on April 27th, 2021. See the published version at https://doi.org/10.1128/mBio.03601-20. 


\section{Abstract}

Background: A ketogenic diet (KD), which is extremely high in fat with a very low carbohydrate level and can induce changes in gut microbiota, has shown benefits for epilepsy and several neurodegenerative diseases. However, the effects of a KD on glucose and lipid metabolism remain inconclusive.

Results: Using two formulas of ketogenic diets (KDR with 89.5\% fat and KDH with 91.3\% fat), which are commonly used in mouse trials, we found that KDR but not KDH induced insulin resistance and damaged glucose homeostasis, while KDH induced more fat accumulation in mice. In addition to the differences in the proportions of nutrients between the two kinds of KD, we found that the sources of fat in KDR were mainly trans-fatty acids. Further study showed that KD impacted glucose metabolism, which was related to the sources of fat, while both the sources and proportions of fat affected lipid metabolism. We found that both the sources and proportions of fat changed the abundance of specific bacteria in Lachnospiraceae and Oscillibacter, which positively correlated with parameters related to lipid accumulation. The abundances of specific bacteria in Bacteroides and Ruminococcaceae were only affected by the sources of fat, and specific bacteria from Lactococcus and Roseburia were only affected by the proportions of fat. Moreover, alterations in the concentrations of SCFAs and tryptophan metabolites, which were negatively correlated with parameters related to lipid accumulation, were also affected by the sources of fat. In addition, the ketogenic diet widely used in human studies still induced insulin resistance and fat accumulation in mice; enhanced Roseburia, Ruminococcaceae and Lachnospiraceae; reduced Turicibacter, increased bile acids and decreased SCFAs.

Conclusions: Overall, our study demonstrated that ketogenic diets induced glucose intolerance and lipid accumulation in mice, which is closely related to the source and proportion of fat in the diet, likely due to changes in the gut microbiota and metabolites.

\section{Background}

Ketogenic diet (KD) refers to an extremely high-fat, very low-carbohydrate diet, which has shown its value in treating epilepsy and its increasing application to other diseases, such as autism spectrum disorder, Alzheimer's disease and metabolic syndrome [1, 2]. However, reports about the effects of a KD on glucose and lipid metabolism in rodents and humans remain inconclusive. Previous clinical trials showed the effectiveness of weight loss and improvement in glucose tolerance and serum lipids in overweight/obese subjects [3-6]. However, a 3-day KD increased postprandial plasma glucose in healthy men [7]. Although it decreased triglycerides, a KD increased cholesterol and inflammatory markers in overweight/obese subjects after 4 weeks of dietary intervention [8]. In a rodent trial, a KD reduced circulating glucose and lipids in both normal weight and ob/ob mouse models [9] and improved glucose tolerance in diet-induced obese mice [10]. Nevertheless, several studies found that a KD has negative effects, including hepatic steatosis, insulin resistance and glucose intolerance [11-17], and a recent study showed that 2-4 months of KD feeding depleted adipose-resident $\gamma \delta T$ cells, which could restrain inflammation, resulting in impaired glucose homeostasis [18]. Although the diets mentioned above are all called a "ketogenic diet", 
the differences in ingredients and dietary composition even in more controlled and standardized animal studies still exist. Therefore, clearly exploring the impact of a KD on glucose and lipid metabolism is needed.

The gut microbiota is critically influenced by diet and has been considered a key mediator linking diet and host physiology [19]. Previous work found that a KD altered the structure of gut microbiota, and specific $\mathrm{KD}$-associated bacteria could protect against seizures in mouse models of intractable epilepsy, which was further confirmed because the protective effects disappeared when mice were treated with antibiotics or reared germ free [20]. Furthermore, a recent study showed that a KD decreased the abundance of Bifidobacterium, and KD-associated gut microbiota reduced the levels of intestinal proinflammatory Th17 cells [21]. Therefore, whether the gut microbiota altered by a KD affects host glucose and lipid metabolism became an interesting question, as did whether microbial-associated metabolites also play a role.

Here, to evaluate the effects of ketogenic diet on host glucose and lipid metabolism, gut microbiota and microbial-associated metabolites, two kinds of ketogenic diets commonly used in mouse trials, type 1 (KDR) and type $2(\mathrm{KDH})$, were investigated in mice. We found that KDR but not $\mathrm{KDH}$ induced insulin resistance and damage to glucose homeostasis, while $\mathrm{KDH}$ induced more fat accumulation in mice, which was associated with their distinct effects on the gut microbiota and metabolite profiles. Moreover, we showed that the differences in metabolic phenotypes in mice induced by these two kinds of KD may be due to the sources and proportions of fat in the diet.

\section{Results}

\section{Ketogenic diet induced glucose intolerance and lipid accumulation in mice}

To observe how two kinds of ketogenic diets impacted physiological and metabolic consequences in mice, 8-week-old male C57BL/6J mice were randomly assigned into four groups: (i) fed ketogenic diet type 1 (KDR); (ii) fed control normal chow of KDR (NCR); (iii) fed ketogenic diet type 2 (KDH); and (iv) fed control normal chow of $\mathrm{KDH}(\mathrm{NCH})$. The proportions of fat were different in these two kinds of ketogenic diets: KDR contained $89.5 \%$ fat, while KDH contained $91.3 \%$ fat. $\beta$-hydroxybutyrate concentrations in blood significantly increased in the mice fed the two kinds of KD, and there was no difference between the KDR and KDH groups (Additional file 1: Figure S1a).

First, we observed how these two kinds of KD affected glucose metabolism. After 5 weeks of dietary intervention, the KDR mice showed significantly higher fasting blood glucose levels than the NCR mice, but there was no significant difference between the mice in the $\mathrm{KDH}$ and $\mathrm{NCH}$ groups (Fig. 1a). Moreover, we found that KDR mice but not KDH mice had slower glucose clearance than their control group during the oral glucose tolerance test (OGTT) (Fig. 1b). Although the secretion of insulin was similar among the four groups of mice, which was confirmed by fasting blood insulin levels and immunofluorescence staining for insulin in the pancreas (Fig. 1c-d), the HOMA-IR index and insulin resistance index were 
significantly increased in the KDR mice (Fig. 1e-g). These results suggested that KDR but not KDH induced insulin resistance and damage to glucose homeostasis in mice.

Next, we observed how these two kinds of KD impacted lipid metabolism. After 5 weeks of dietary intervention, the KDH mice had lower body weights (Additional file 1: Figure S1b-c) and higher weights of epididymal and retroperitoneal fat mass (Fig. $1 \mathrm{~h}$-i) than the $\mathrm{NCH}$ mice. Furthermore, the KDH mice also exhibited excessive lipid accumulation in the liver with increased concentrations of liver total cholesterol and triglyceride compared to the $\mathrm{NCH}$ mice (Fig. 1j-k). However, there was no significant difference in the above parameters between the mice in the KDR and NCR groups. Both the adipocyte size and NAFLD activity score significantly increased in the mice fed the two kinds of KD compared to their respective control groups, which were estimated by hematoxylin and eosin (H\&E)-stained epididymal fat and liver, and the KDH mice had significantly higher values than the KDR mice (Fig. 1I-m). These results suggested that $\mathrm{KDH}$ induced more fat accumulation in mice than KDR.

Previous studies have shown that metabolic diseases, including type 2 diabetes (T2D) and nonalcoholic fatty liver disease (NAFLD), which disrupt glucose and lipid metabolism, are characterized by an impaired and defective intestinal barrier [22]. To determine whether intestinal permeability was impacted by two kinds of KD, we measured the expression of Zo-1, Occludin, Muc1, Muc2 and Muc3 in the colon. The KDR and KDH mice showed significantly lower expression of Occludin and Muc2 than their control groups, implying that the intestinal barrier was damaged in the mice fed with the two kinds of KD (Additional file 1: Figure S2a), which was further confirmed by immunohistochemistry staining for ZO-1 in the ileum (Additional file 1: Figure S2b). Moreover, we found that the serum level of lipopolysaccharide (LPS)binding protein (LBP) was significantly enhanced in the mice fed with the two kinds of KD, implying increased intestinal permeability to LPS, and there was no difference between KDR and KDH mice (Additional file 1: Figure S2c). These results suggested that both KDR and KDH impaired intestinal barrier function in mice.

\section{Two kinds of ketogenic diets molded disparate gut microbiota in mice}

By qPCR targeting the 16S rRNA gene, we found that the total bacterial load in feces was similar among the four groups of mice (Additional file 1: Figure S3). To analyze whether the structure of gut microbiota was modulated by these two kinds of ketogenic diets, we sequenced the 16S rRNA V3-V4 region of fecal samples collected at the $5^{\text {th }}$ week. After 5 weeks of intervention, the richness and diversity of gut microbiota, which was reflected by the numbers of observed amplicon sequence variants (ASVs) and the Shannon index, were significantly increased only in the KDH mice but not in the KDR mice compared to their control groups (Additional file 1: Figure S4). Principal coordinate analysis (PCoA) and permutational multivariate analysis of variance (PerMANOVA) based on Bray-Curtis distances showed clear separations of gut microbiota structure between the mice in the KDR and NCR groups, as well as the KDH and NCH groups ( $P<0.01$ with PerMANOVA, 999 permutations). Although the gut microbiota structure was more similar in the mice fed the two kinds of KD than in mice fed the two control diets, there was still a 
significant difference between the KDR and KDH mice (Fig. 2a-b). Thus, these two types of KD induced different changes in the gut microbiota in mice.

We constructed sparse partial least-squares discriminant analysis (sPLS-DA) models to identify members of the gut microbiota responding to these two kinds of KD intervention. We found that 44 ASVs were shifted in the KDR mice compared to the NCR mice, while 40 ASVs showed differences between the KDH and NCH mice (Additional file 1: Figure S5-6, Additional file 2: Table S1). Notably, only 16 ASVs showed the same changes in both the KDR and KDH mice. Of these, the abundances of ASVs, including Ruminococcaceae (ASV208, ASV291), Intestinimonas (ASV43, ASV96, ASV272), and Lachnospiraceae (ASV44, ASV18, ASV159, ASV164, ASV165), were significantly enriched by both types of KD, and all these ASVs positively correlated with parameters related to glucose intolerance and lipid accumulation. Furthermore, 6 ASVs, including Enterococcus (ASV5), Blautia (ASV31), Turicibacter(ASV38), Lachnoclostridium (ASV56) and Lachnospiraceae (ASV59, ASV65), were significantly decreased by both $\mathrm{KDs}$, and all these ASVs negatively correlated with parameters related to glucose intolerance and lipid accumulation. Next, we compared the gut microbiota in mice with the two types of KD and identified 38 significantly different ASVs (Additional file 1: Figure S7, Additional file 2: Table S1). Among these 38 ASVs, Bacteroides (ASV8, ASV36, ASV42), Lachnospiraceae (ASV313) and Ruminococcaceae (ASV60, ASV73) were significantly enriched in the KDR mice and only positively correlated with parameters related to glucose intolerance. Moreover, Roseburia (ASV9, ASV63), Negativibacillus (ASV112), Acetatifactor (ASV22), Oscillibacter (ASV202), Lachnoclostridium (ASV285) and Lachnospiraceae (ASV25, ASV157, ASV159, ASV160, ASV169, ASV279, ASV286, ASV316) were significantly increased in the KDH mice and only positively correlated with parameters related to lipid accumulation (Fig. 2c). Taken together, the results suggested that two types of KD introduced different changes in the gut microbiota.

\section{Changes in the metabolome in mice fed two kinds of ketogenic diets}

To identify how these two kinds of KD affected metabolites of the gut microbiota in mice, the untargeted metabolome of the cecum content was analyzed by liquid chromatography-mass spectrometry (LC-MS), and principal component analysis (PCA) showed that the metabolome profiles of the gut microbiota in the $\mathrm{KDR}$ and $\mathrm{KDH}$ mice were significantly different from those of their control groups. Moreover, the metabolite profiles of the KDR and KDH mice clearly segregated in the first principal component (Fig. 3a). Hence, KD intervention changed the metabolites in the gut, and the types of ketogenic diets had different influences.

From orthogonal projection to latent structure-discriminant analysis (OPLS-DA) models (Additional file 1: Figure S8), we identified differential metabolic features $(\mathrm{VIP}>1, P<0.05)$ that included 127 metabolites between the KDR and NCR mice, 125 metabolites between the KDH and NCH mice and 117 metabolites between the KDH and KDR mice (Additional file 2: Table S2). A total of 156 of these metabolites were significantly correlated with host parameters related to glucose and lipid metabolism (Additional file 2: Table S3). In addition, 85 of these 156 metabolites were significantly correlated with gut microbial variations ( $\mathrm{R}^{2}>0.09, P<0.05$ of PerMANOVA) (Additional file 2: Table S3). Among these 85 metabolites, 
suberic acid was significantly enhanced in both the KDR and KDH mice, and it positively correlated with parameters related to glucose intolerance and lipid accumulation. Moreover, nervonic acid and batyl alcohol, which were significantly decreased by both KDs, were negatively correlated with parameters related to glucose intolerance and lipid accumulation. Deoxycholic acid, chenodeoxycholate, and trans-2-hydroxycinnamic acid, which only increased in the KDR mice, were positively correlated with glucose intolerance. Indoleacetic acid and isobutyric acid, which only decreased in the KDH mice, were negatively correlated with lipid accumulation (Fig. 3b-c). Taken together, the results suggested that the mice had significantly different metabolite profiles between the KDR and KDH mice.

\section{The sources and proportions of fat in the ketogenic diet affected the metabolic phenotypes in mice}

The two kinds of KD induced different damage to metabolic phenotypes in the mice, which was associated with gut microbiota and metabolite changes, although the components of macronutrients were virtually identical in the KDR and KDH. Then, we customized a ketogenic diet called KD(89.5\%) with the same proportions of fat as KDR and the same sources as KDH in nutrients to evaluate the influences of sources and proportions of fat in the ketogenic diets on the metabolic phenotypes in mice. We obtained comparable results for parameters related to glucose and lipid metabolism for the mice fed KDH and $\mathrm{NCH}$ to those in the abovementioned trial. Unlike the mice in the KDR group, which had dramatic damage to glucose metabolism, the KD(89.5\%) mice had fasting blood glucose levels, glucose clearance and insulin resistance indices similar to the KDH mice (Fig. 4a-f). Furthermore, the KD(89.5\%) mice exhibited an increase in epididymal and retroperitoneal fat mass compared to the $\mathrm{NCH}$ mice (Fig. 4g-i), which was not observed between the mice in the KDR and NCR groups. Interestingly, the KD(89.5\%) mice did not develop excessive lipid accumulation in the liver like the KDH mice (Fig. 4j-I), indicating that only $\mathrm{KDH}$, which contained $91.3 \%$ fat, induced lipid accumulation in the livers of mice. Taken together, these results suggested that the sources but not proportions of fat in the ketogenic diet induced differences in metabolic phenotypes related to glucose metabolism, while both the sources and proportions induced differences in metabolic phenotypes related to lipid metabolism.

Based on the above results, we concluded that the sources of fat in the ketogenic diet had important and significantly different effects on metabolic phenotypes in mice. Therefore, we quantified the concentrations of fatty acids in the three different ketogenic diets (KDR, KDH and $K D(89.5 \%)$ ) and found that trans-fatty acids were dramatically increased in $K D R$, which was confirmed by the concentrations of tran-6-octadecenoic acid, tran-9-octadecenoic acid and tran-11-octadecenoic acid, and there was no significant difference between KDH and KD(89.5\%) (Additional file 1: Figure S9). Combining the results that KDR but not KDH induced insulin resistance and damaged glucose homeostasis in mice, we speculated that the trans-fatty acids in KDR may be an important suspect.

\section{The gut bacteria and metabolites affected by the sources and proportions of fat in ketogenic diets}

By 16S rRNA gene sequencing, we found that there were still significant differences in the gut microbiota between the mice in the KDH and $\mathrm{KD}(89.5 \%)$ groups ( $P<0.05$ with PerMANOVA, 999 permutations) (Fig. $5 a)$. Next, we observed how the abundances of 87 ASVs affected by two kinds of KD in the 
abovementioned trial changed. Of the mice fed these two ketogenic diets with the same proportion but different sources of fat, the KD(89.5\%) mice had an almost negligible abundance of Bacteroides (ASV8, ASV36, ASV42) and Ruminococcaceae (ASV60, ASV73), similar to the NCH mice but unlike the KDR mice, which showed significant enrichment in these microbes. However, the $\mathrm{KD}(89.5 \%)$ mice exhibited a significant increase in the abundance of Lachnospiraceae (ASV25, ASV160, ASV169), Intestinimonas (ASV96) and Oscillibacter (ASV202), and there was no significant difference between the KDR and NCR mice (Fig. 5b-c). In addition, for these two ketogenic diets with the same source but different proportions of fat, although the abundance of most ASVs was similar between the KDH and $\mathrm{KD}(89.5 \%)$ mice, differences still existed. KD(89.5\%) significantly increased the abundance of ASVs, including ASV25 (Lachnospiraceae), ASV26 (Lactococcus) and ASV63 (Roseburia), and decreased the abundance of ASV202 (Oscillibacter) compared to the KDH mice (Fig. 5d-e). Taken together, we identified 10 ASVs and 4 ASVs that responded to the sources and proportions of fat in ketogenic diets, respectively. Thus, these 14 ASVs were considered key members that were affected by the sources and proportions of fat in the two kinds of ketogenic diets.

We also observed how the metabolites of the gut microbiota affected by the two kinds of ketogenic diets in the abovementioned trial changed, including short-chain fatty acids (SCFAs), bile acids (BAs) and tryptophan metabolites. The results we obtained for the $\mathrm{KDH}$ mice compared to the $\mathrm{NCH}$ mice were comparable to those in the abovementioned trial. Of note, the $\mathrm{KD}(89.5 \%)$ mice also exhibited a significant decrease in the concentrations of total SCFAs and tryptophan metabolites compared to the NCH mice, which were not observed between the KDR and NCR mice (Fig. 5f-g). Furthermore, only the KDR mice exhibited dramatically increased BA levels in untargeted metabolome analysis; however, targeted bile acid quantitation showed that the $\mathrm{KDH}$ and $\mathrm{KD}(89.5 \%)$ mice also had increased secondary BA levels (Fig. 5h), with significant differences compared to the $\mathrm{NCH}$ mice. These results suggested that the sources of fat in the ketogenic diet introduced different changes in metabolite profiles, despite slight differences in the concentration of metabolites in different fat proportions.

\section{The ketogenic diet used in human studies still impaired metabolic health, gut microbiota and metabolites in mice}

Considering that the ketogenic diets used in the above two trials were nearly devoid of carbohydrates, the ketogenic diets used in human studies permit low carbohydrate consumption (approximately $5 \%$ to $10 \%$ of total caloric intake or below $50 \mathrm{~g}$ per day) [19]. Thus, we customized another ketogenic diet called $\mathrm{KD}(72 \%)$ with $72 \%$ fat, $20 \%$ protein and $8 \%$ carbohydrate. Notably, to eliminate the influence of trans-fatty acids of diet, $\mathrm{KD}(72 \%)$ used the same sources of nutrients as $\mathrm{KDH}$. We observed increased $\beta$ hydroxybutyrate concentrations in the $\mathrm{KD}(72 \%)$ mice (Additional file 1: Figure S10). Moreover, $\mathrm{KD}(72 \%)$ damaged metabolic health, which was mainly reflected in two aspects. First, although the fasting blood glucose level and glucose clearance during OGTT in the $\mathrm{KD}(72 \%)$ mice were similar to those in the NC group (Fig. 6a-b), the secretion of insulin was significantly higher in the KD(72\%) mice (Fig. 6c-d), and the $\mathrm{KD}(72 \%)$ mice also had a higher HOMA-IR index and insulin resistance index (Fig. 6e-f). This suggests that insulin sensitivity was reduced in the $\mathrm{KD}(72 \%)$ mice. Second, the $\mathrm{KD}(72 \%)$ mice showed significantly 
higher fat mass and adipocyte size than the NC mice (Fig. $6 \mathrm{~g}-\mathrm{h}$ ). To summarize, these results showed that $\mathrm{KD}(72 \%)$ induced insulin resistance and fat accumulation in mice.

By $16 \mathrm{~S}$ rRNA gene sequencing, we found that $\mathrm{KD}(72 \%)$ group displayed significantly altered gut microbiota compared to the NC group ( $P<0.01$ with PerMANOVA, 999 permutations) (Fig. 7a). Although selected specific ASVs were not exactly the same (Additional file 1: Figure S11, Additional file 2: Table S4), KD(72\%) still enhanced the abundance of Roseburia, Ruminococcaceae, and Lachnospiraceae and reduced the abundance of Turicibacter similar to the diets in the abovementioned trials (Fig. 7b). Furthermore, significantly decreased SCFAs and increased BA levels were also observed in the KD $(72 \%)$ mice (Fig. 7c-d). Although the concentrations of 3-indoleacrylic acid were significantly decreased in the $\mathrm{KD}(72 \%)$ mice, there was no significant difference in the concentrations of other tryptophan metabolites compared to those in the NC group (Fig. 7e). Taken together, these results suggested that $\operatorname{KD}(72 \%)$ impaired metabolic health, gut microbiota and metabolites in mice.

\section{Discussion}

In the current study, we showed that the impaired glucose and lipid metabolism in mice induced by ketogenic diets may be due to the sources and proportions of fat in these diets, which are associated with their distinct effects on gut microbiota and metabolites.

The ketogenic diets, regardless of the proportions and sources of fat, impaired glucose and lipid metabolism in mice in our study, which is in agreement with previous reports that KDs caused glucose intolerance, insulin resistance, hepatic steatosis and fat accumulation [11-17]. A KD induced glucose intolerance and insulin resistance, which may be due to the reduction in $\gamma \delta \mathrm{T}$ cells that can restrain inflammation [18]. Bielohuby et al suggested that the effect of a KD on glucose and insulin resistance would be caused by the decreased cell surface expression of GLUT4 in skeletal muscle, which reduces glucose uptake in the periphery [11]. Regarding the hepatic lipid accumulation induced by a KD, Crawford et al reported that the synergistic effects of protein restriction and choline deficiency may be potential contributors [23]. Notably, an impaired colonic mucus barrier has been suggested as one of the causes of metabolic damage [24], and the expression of genes related to the intestinal barrier (Occludin, Muc2) was significantly reduced by 5 weeks of KD intervention in our work. Future studies are still required to further investigate the overall mechanism by which KDs impair glucose and lipid metabolism.

Our study highlights the potential role of the gut microbiota and metabolites in the induction of glucose intolerance and lipid accumulation in response to KD consumption. We reported changes in the gut microbiota after KD intervention, with increased levels of Ruminococcaceae and Lachnospiraceae, which were positively correlated with glucose intolerance and lipid accumulation. However, a recent study showed that the abundance of Ruminococcaceae and Lachnospiraceae decreased after changing a baseline diet to a ketogenic diet [21]. This discrepancy may have occurred because the experimental subjects in our study were normal mice, whereas those in other studies were overweight or obese people. Furthermore, we found that metabolites of gut microbiota, such as SCFAs, tryptophan metabolites and 
BAs, were related to $\mathrm{KD}$-induced metabolic damage. The mechanism may be that reduced SCFAs and tryptophan metabolites, as well as increased BAs, can promote intestinal permeability [25], allow excessive LPS into circulation, stimulate the release of proinflammatory cytokines, and result in impaired glucose and lipid metabolism [26]. In addition, the two types of KD introduced different changes in metabolites. KDR dramatically increased BA levels. It is known that high-fat diet intake stimulates the secretion of bile acids and increases fecal concentrations of secondary BAs [27], which can cause colorectal and hepatic carcinogenesis via the induction of necrosis with inflammation [28]. KDH significantly decreased the levels of SCFAs, which can improve metabolic diseases by HDAC inhibition [29] and GPR41/43 activation [30]. Moreover, KDH also reduced tryptophan metabolites, which can activate the aryl hydrocarbon receptor (AHR) [31], promote anti-inflammatory signals and ameliorate metabolic syndrome. Regarding the different metabolic phenotypes induced by these two types of KD, the mechanism involved still needs further investigation.

More importantly, we showed that both the sources and proportions of fat in the ketogenic diets modified glucose and lipid metabolism in mice, suggesting that the controversial results from previous reports may be due to neglect of the composition of the ketogenic diet. We found that KDR but not KDH induced glucose intolerance and insulin resistance. A recent study using the same ketogenic diet (D12369B) as KDR in our experiments demonstrated that long-term KD feeding depleted adipose-resident $\gamma \delta \mathrm{T}$ cells, leading to obesity and the loss of glycemic regulation in mice [18]. Other studies using a diet consistent with our KDH showed reduced circulating glucose in animal models [20,32]. In our study, we found that the concentrations of trans-fatty acids (TFA) dramatically increased in KDR, which was associated with an increased risk of cardiovascular disease and incidence of obesity and type 2 diabetes mellitus [33, 34]. These data suggest that glucose intolerance and insulin resistance induced by KDR may be the consequence of high levels of TFA. Thus, future work related to diet should not only consider the proportion of nutrients, but the source should also be regarded as critical. These factors may have a considerable impact on the experimental results, especially in metabolism.

\section{Conclusions}

Overall, our study demonstrates that ketogenic diets induced glucose intolerance and lipid accumulation in mice, which is closely related to the sources and proportions of fat in the diet, and these effects were likely due to changes in the gut microbiota and metabolites. Although KDs have beneficial effects on epilepsy and neurodegenerative diseases [1], according to our findings, KDs truly impair glucose and lipid metabolism. Thus, when using these diets for weight loss and the treatment of other conditions, the risk of glucose and lipid metabolism disorders should be kept in mind, and the sources and proportions of fat in these diets need to be further evaluated.

\section{Methods}

\section{Animal trial}


All animal experimental procedures were approved by the Institutional Animal Care and Use Committee (IACUC) of Shanghai Jiao Tong University (no. 2018043 \& 2018106). The mice were purchased from SLAC, Inc. (Shanghai, China), and the experiments were performed at the animal center of Shanghai Jiao Tong University. All mice were kept under a standard $12 \mathrm{~h}$ light/dark cycle (lights on at 7:00 am) and a temperature of $22^{\circ} \mathrm{C} \pm 3^{\circ} \mathrm{C}$.

Trial 1 After two weeks of accommodation, 8-week-old specific pathogen-free (SPF) C57BL/6J male were randomly assigned into four groups ( 9 mice per group) and fed two kinds of ketogenic diets or their control normal chow for 5 weeks: (i) fed ketogenic diet type 1(KDR; Research Diets D12369B, 0.1\% carbohydrate, $10.4 \%$ protein, $89.5 \%$ fat, $6.76 \mathrm{kcal} / \mathrm{g}$ ); (ii) fed control normal chow of KDR (NCR; Research Diets D12450J; $70 \%$ carbohydrate, $20 \%$ protein, $10 \%$ fat, $3.85 \mathrm{kcal} / \mathrm{g}$ ); (iii) fed ketogenic diet type 2 (KDH; $1 \%$ carbohydrate, $7.7 \%$ protein, $91.3 \%$ fat, $6.77 \mathrm{kcal} / \mathrm{g}$, produced by FBSH Biotechnology Ltd., Shanghai, China); or (iv) fed control normal chow of $\mathrm{KDH}(\mathrm{NCH} ; 64.5 \%$ carbohydrate, $20 \%$ protein, $15.5 \%$ fat, 4 $\mathrm{kcal} / \mathrm{g}$, produced by FBSH Biotechnology Ltd., Shanghai, China). The formulas of these diets were shown in Additional file 3. The mice were allowed ad libitum access to water and food. Both the KDR and KDH mice were fed freshly prepared ketogenic diets every day because of the perishability of ketogenic diets. The body weight of the mice and food intake per cage (3 mice per cage) were measured every three days.

Trial 2 After two weeks of acclimation, 8-week-old specific pathogen-free (SPF) C57BL/6J males were randomly assigned into three groups (9 mice per group) and fed ketogenic diets with two different proportions of fat or normal chow for 5 weeks: (i) fed normal chow (NCH); (ii) fed KDH; or (iii) fed a ketogenic diet containing $89.5 \%$ fat (KD(89.5\%); $0.1 \%$ carbohydrate, $10.4 \%$ protein, $89.5 \%$ fat, $6.57 \mathrm{kcal} / \mathrm{g}$; produced by FBSH Biotechnology Ltd., Shanghai, China). The formulas of these diets were shown in Additional file 3. The mice were allowed ad libitum access to water and food. Both the KDH and $\mathrm{KD}(89.5 \%)$ mice were fed freshly prepared ketogenic diets every day. The body weight of the mice and food intake per cage (3 mice per cage) were measured every three days.

Trial 3 After two weeks of accommodation, 8-week-old specific pathogen-free (SPF) C57BL/6J males were randomly assigned into two groups (9 mice per group) and fed a ketogenic diet or normal chow for 5 weeks: (i) fed normal chow (NC; same as $\mathrm{NCH}$ in the abovementioned trial) or (ii) fed a ketogenic diet containing $72 \%$ fat (KD(72\%); $8 \%$ carbohydrate, $20 \%$ protein, $72 \%$ fat, $5.65 \mathrm{kcal} / \mathrm{g}$; produced by FBSH Biotechnology Ltd., Shanghai, China). The formulas of these diets were shown in Additional file 3. The mice were allowed ad libitum access to water and food. The $\mathrm{KD}(72 \%)$ mice were fed a freshly prepared ketogenic diet every day. The body weight of the mice and food intake per cage ( 3 mice per cage) were measured every three days.

\section{Histopathology of epididymal adipose tissue, liver, pancreas and ileum}

For epididymal adipose tissue (eAT) and liver, fresh tissue was fixed with $4 \%$ paraformaldehyde, embedded in paraffin, sectioned (4- $\mu \mathrm{m}$ thickness) and stained with hematoxylin and eosin (H\&E) (Wuhan Servicebio Technology Ltd., Wuhan, China). Adipocyte size of eAT sections was assessed using ImagePro Plus v6.0 (Media Cybernetics Inc., Silver Springs, MD, USA), and the mean cell area of adipocytes was 
determined in at least three discontinuous scans (> 300 total adipocytes) in five random mice from each group. The histologic scores (NAFLD Activity Score) of liver sections were assessed as described previously [35]. For the pancreas, fresh tissue was fixed with $4 \%$ paraformaldehyde, embedded in paraffin, sectioned (4- $\mu \mathrm{m}$ thickness) and stained for insulin (green) after standard indirect immunofluorescence staining. The islet areas were determined in at least three discontinuous scans under $400 x$ magnification and examined as the total islet areas divided by the total number of islets. For the ileum, immunohistochemistry staining for ZO-1 was performed as previously described [36].

\section{Blood ketone measurement}

After $6 \mathrm{~h}$ of fasting, blood ketone ( $\beta$-hydroxybutyrate) levels were measured in blood samples collected from the tip of the tail vein with a blood ketone meter (FreeStyle Optium Neo, Abbott, USA).

\section{Oral Glucose Tolerance Test}

After $6 \mathrm{~h}$ of fasting, mice were administered glucose ( $2 \mathrm{~g} / \mathrm{kg}$ body weight) by oral gavage, and blood samples were collected from the tip of the tail vein at $0,15,30,60,90$ and $120 \mathrm{~min}$ after glucose administration for assessment of glucose concentrations using a blood glucose meter (Accu-Chek ${ }^{\circledR}$ Performa, Roche, USA). Blood samples were collected from the tail vein into tubes at 0,15 , and $60 \mathrm{~min}$ after glucose administration to determine insulin concentrations. The degree of insulin resistance was estimated by calculating the HOMA-IR (homeostatic model assessment for insulin resistance, glucose $x$ insulin/22.5*21.2 from fasting samples) index and insulin resistance index (the product of the 0-60 min area under curve (AUC) of blood glucose and that of serum insulin in OGTT).

\section{Serum LBP and insulin measurement}

After blood was placed at room temperature for $40 \mathrm{~min}$, the samples were centrifuged at 3,500 rpm for 15 min at $4^{\circ} \mathrm{C}$, and the supernatant was collected and then stored at $-80^{\circ} \mathrm{C}$ until further analyses. According to the manufacturer's instructions, enzyme-linked immunosorbent assays (ELISAs) were used to quantify lipopolysaccharide (LPS)-binding protein (LBP) (PCDBM0177; P\&C Biotechnology Ltd., China) and insulin (90080; Chrystal Chem, USA).

\section{Serum and hepatic lipid measurement}

Assay kits were used to measure concentrations of serum total cholesterol (A111-1-1; Nanjing Jiancheng Bioengineering Institute, Nanjing, China) and triglyceride (A110-1-1; Nanjing Jiancheng Bioengineering Institute, Nanjing, China). Semi-thawed liver tissue samples were cut to approximately $0.1 \mathrm{~g}$ and homogenized in a corresponding volume (W/V:1/9) of homogenizing buffer $(\mathrm{pH} 7.4 ; 0.01 \mathrm{~mol} / \mathrm{L} \mathrm{Tris-HCl,}$ $0.1 \mathrm{mmol} / \mathrm{L}$ EDTA-2Na, $0.8 \% \mathrm{NaCl}$ ) in screw tubes. Next, the tubes were shaken for 5 min at $25 \mathrm{~Hz} / \mathrm{s}$ with a Tissuelyser (QIAGEN, Germany) and then put into an ice water mixture for $5 \mathrm{~min}$, and the above steps were repeated again. Afterwards, the supernatant was collected after being centrifuged at 2,500 rpm for $10 \mathrm{~min}$ at $4^{\circ} \mathrm{C}$ and used to measure concentrations of hepatic total cholesterol and triglyceride using the 
same kits described above. The hepatic lipid results were corrected by calculating the total protein concentration (20202ES76; Yeasen Biotechnology Ltd., China).

\section{Colon RNA isolation and reverse transcription qPCR (RT-qPCR)}

According to the manufacturer's protocol, total RNA was extracted from the colon using an RNeasy Minikit (74804; Qiagen, Germany) and reverse transcribed into cDNA using a SuperScriptTM kit (18080051; Invitrogen, USA). Quantitative real-time PCR (qPCR) was performed in an $18 \mu$ reaction system containing forward and reverse primers and SYBR Green I PCR Supermix (Bio-Rad) on a LightCycler 96 system (Roche Applied Sciences) and used to assess the expression of Zo-1, Occludin, Muc1, Muc2 and Muc3. The PCR conditions were $95^{\circ} \mathrm{C}$ for $3 \mathrm{~min}$, followed by 40 cycles of $95^{\circ} \mathrm{C}$ for $20 \mathrm{~s}, 56^{\circ} \mathrm{C}$ for $30 \mathrm{~s}$, and $72^{\circ} \mathrm{C}$ for $30 \mathrm{~s}$, and plate reads for $5 \mathrm{~s}$. Gene expression levels were determined using the $2^{-\triangle \Delta C T}$ method, with Beta-actin serving as the reference gene. Forward $(F)$ and reverse $(R)$ primer sequences [37] are as follows: Zo-1, F-ACCCGAAACTGATGCTGTGGATAG, and R-AAATGGCCGGGCAGAACTTGTGTA; Occludin, F-ATGTCCGGCCGATGCTCTC and R-TTTGGCTGCTCTTGGGTCTGTAT; and Muc1, F-

TACCCTACCTACCACACTCACG and R-CTGCTACTGCCATTACCTGC; MUC2, F-CACCAACACGTCAAAAATCG and R-GGTCTCTCGATCACCACCAT; MUC3, F-CTTCCAGCCTTCCCTAAACC and R-

TCCACAGATCCATGCAAAAC; Beta-actin, F-GGCTGTATTCCCCTCCATCG and RCCAGTTGGTAACAATGCCATGT.

\section{Statistical Analysis for Animal Trials}

Statistical significance for physiological and biochemical data of mice in different groups was analyzed with one-way analysis of variance (ANOVA) followed by a Tukey post hoc test using the "stats" and "multcomp" packages in R (v3.6.1) (www.r-project.org). Differences were considered statistically significant when the $P$ value was $<0.05$.

\section{qPCR of total fecal bacteria}

A plasmid of the $16 \mathrm{~S}$ full-length positive Akkermansia muciniphila strain $\left(\mathrm{N}=10^{9} \mathrm{copies} / \mu \mathrm{L}\right)$ was diluted according to different gradients successively to $10^{8}, 10^{7}, 10^{6}, 10^{5}, 10^{4}, 10^{3}$, and $10^{2}$ copies/ $\mu \mathrm{L}$. qPCR was performed in a $20 \mu \mathrm{L}$ reaction system containing template $(20 \mathrm{ng})$, primer Uni331F (5'-

TCCTACGGGAGGCAGCAGT-3'), primer Uni797R (5'-GGACTACCAGGGTATCTAATCCTGTT-3') [38] and supermix (Bio-Rad) on a LightCycler 96 system, with 2 replicates for standard and sample DNA. The PCR conditions were $95^{\circ} \mathrm{C}$ for $3 \mathrm{~min}$, followed by 40 cycles of $95^{\circ} \mathrm{C}$ for $15 \mathrm{~s}, 60^{\circ} \mathrm{C}$ for $60 \mathrm{~s}$, and $80^{\circ} \mathrm{C}$ for $5 \mathrm{~s}$, and plate reads for $5 \mathrm{~s}$. A standard curve was determined though a linear fit of the copy number and CT value of the plasmid in different gradients. The copy number of sample DNA was calculated though a standard curve using Opticon Monitor 3.1. Data were analyzed with one-way ANOVA followed by a Tukey post hoc test. 
During the animal trial, fresh feces were collected weekly and stored at $-80^{\circ} \mathrm{C}$ until analysis. DNA was extracted from fecal samples collected at the $5^{\text {th }}$ week using a previously described method [39]. A sequencing library of the V3-V4 regions of the 16S rRNA gene was constructed according to the manufacturer's instructions (Part \# 15044223 Rev. B; Illumina Inc., CA, USA) with some modifications as previously published [40] and sequenced on the MiSeq system (Illumina, Inc., CA, USA) with the MiSeq reagent kit v3 $(2$ x 300 cycles, no. MS-102-3033).

The raw paired-end reads were submitted to Quantitative Insights Into Microbial Ecology2 (QIIME2, v 2018.11) [41] for analysis. Sequence quality and primer removal were processed using the "Demux" and "cutadapt" plugins, respectively, and DADA2 [42] was used to obtain quality filtered, denoised, chimerafree and merged amplicon sequence variant (ASV) [43]. Representative sequences for each ASV were built into a phylogenetic tree by FastTree and assigned taxonomic classifications using the SILVA132 16S rRNA database [44]. Alpha diversity metrics (observed ASVs and Shannon index), beta diversity metrics and principal coordinate analysis (PCOA) were calculated using the "core-metrics-phylogenetic" plugin after sequencing depth was downsized to 20,000 (Trail 1) and 13,000 (Trail 2, 3) sequences per sample. The statistical significance of gut microbiota among different groups was assessed by permutational multivariate analysis of variance (PERMANOVA; 999 permutations). PERMANOVA clustering analysis was performed with Bray-Curtis distances. Differences were considered significant when $Q<0.05$.

Sparse partial least-squares discriminant analysis (sPLS-DA) [45] was performed using the "mixOmics" (v6.8.2) package [46] in R to identify the discriminative ASVs between different groups (NCR \& KDR, NCH $\& \mathrm{KDH}, \mathrm{KDR} \& \mathrm{KDH})$. The optimal classification performance of the sPLS-DA model was assessed with the perf function using leave-one-out cross-validation with the smallest error rate. Variations in the relative abundance of selected ASVs in different groups were analyzed by the Mann-Whitney $U$ test in MATLAB (R2017b). Differences were considered significant when $P<0.05$.

The visual presentation of the heatmap showing the relative abundance of selected ASVs in different groups was performed using the "pheatmap" package in R. Spearman correlations between ASV abundance and host parameters related to glucose and lipid metabolism were calculated using MATLAB. The $P$-values were adjusted by false discovery rate (FDR) estimation using the Benjamini-Hochberg method [47]. The visual presentation of correlations was performed using the "ggcorrplot" package in R. Correlations were considered significant when FDR $<0.05$.

\section{Untargeted metabolomics study and data analysis}

Cecum content samples $(60 \mathrm{mg}$ ) were homogenized in $200 \mu \mathrm{L}$ distilled water and vortexed. Next, $800 \mu \mathrm{L}$ of methanol:acetonitrile solution $(1: 1, \mathrm{v} / \mathrm{v})$ mixture was added to precipitate protein, pulverized by ultrasonic wave at low temperature for $30 \mathrm{~min}$ and then kept at $-20^{\circ} \mathrm{C}$ for $1 \mathrm{~h}$. Next, samples were centrifuged at $14,000 \mathrm{rcf}$ for $20 \mathrm{~min}$ at $4{ }^{\circ} \mathrm{C}$ and stored at $-80^{\circ} \mathrm{C}$ after removing the supernatant and vacuum drying. Quality control (QC) samples were prepared using the same methods. HPLC-Q-TOF MS analysis of cecal contents in mice was performed by Shanghai Applied Protein Technology Ltd. 
(Shanghai, China) on the 1290 Infinity Ultrahigh Performance Liquid Chromatography system (Agilent, Germany) coupled with a Triple TOF 5600 system (AB SCIEX, USA). The raw data were converted into $\mathrm{mzXML}$ format using Proteo Wizard software, and peak alignment, retention time correction and peak area extraction were performed using the XCMS program. Details of the data processing and analysis were performed as described previously [48].

Variable importance in projection (VIP) scores obtained from the orthogonal projection to latent structurediscriminant analysis (OPLS-DA) model were used to assess the contribution of variables. Metabolites that had VIP > 1 between different groups (NCR \& KDR, NCH \& KDH, KDR \& KDH) were defined as discriminating metabolites, and then these discriminating metabolite levels were compared between different groups using Student's t test statistical analysis. Only discriminating metabolites that had $P<$ 0.05 were defined as significant discriminative metabolites and identified by searching an in-house standard MS/MS library (using exact mass data (mass error $\leq 25 \mathrm{ppm}$ ) or MS/MS spectra matching).

The visual presentation of the heatmap showing the standardized concentration of significant discriminative metabolites in different groups was performed using the "pheatmap" package in R. Spearman correlations between metabolite concentration and host parameters related to glucose and lipid metabolism were calculated using MATLAB. The $P$-values were adjusted by FDR estimation. The visual presentation of correlations was performed using the "ggcorrplot" package in R. Correlations were considered significant with an FDR $<0.05$.

The "effect size" analysis strategy [49] was used to determine the biomarker metabolites affecting the gut microbiome. The adonis function of the "vegan" package in R was used to estimate the "one-to-all" effect size $\left(R^{2}\right)$ between each single variable of metabolites to the whole gut microbiome. Only variables with significant $\left(P<0.05,999\right.$ permutations) effects and $\mathrm{R}^{2}>0.09$ on the gut microbiome were considered later.

\section{Targeted fatty acid quantitation}

GS-MS analysis of fatty acids in the three ketogenic diets (KDR, KDH, KD (89.5\%); 3 replicates for each diet) was performed by BioNovoGene Technology Ltd. (Suzhou, China) on a GS-MS-QP2010 Ultra system (Shimadzu, Japan). Data were analyzed with one-way ANOVA followed by a Tukey post hoc test. Samples (200 400 mg) were homogenized in a $1 \mathrm{~mL}$ N-hexane:isopropanol solution (3:2, v/v) mixture with $3 \mathrm{~mm}$ diameter zirconia/silica steel beads, pulverized by ultrasonication for $30 \mathrm{~min}$ and then centrifuged at 12,000 rpm for $5 \mathrm{~min}$. Then, $500 \mu \mathrm{L}$ of supernatant was added to $1 \mathrm{~mL} \mathrm{~N}$-hexane. After repeating centrifugation for $5 \mathrm{~min}, 900 \mu \mathrm{L}$ of supernatant was added to $400 \mu \mathrm{L}$ methanol. After pulverizing in an ultrasonic wave for $5 \mathrm{~min}, 40 \mu \mathrm{L}$ diazomethane was added and kept at room temperature for $15 \mathrm{~min}$, and then centrifugation was repeated. $200 \mu \mathrm{L}$ of $\mathrm{N}$-hexane was added after collecting the supernatant $(200 \mu \mathrm{L})$ and vacuum drying, and the samples were centrifuged. Finally, the supernatant was collected and transferred into a GC vial for GC-MS analysis.

\section{Targeted short-chain fatty acid (SCFA) quantitation}


GS-MS analysis of SCFAs in the cecal contents of mice was performed on an Agilent 6890 (Agilent Technologies, CA, USA) with flame ionization, thermal conductivity detectors and capillary columns. Data were analyzed with one-way ANOVA followed by a Tukey post hoc test. Then, $50 \mathrm{mg}$ cecal contents was homogenized with $250 \mu \mathrm{L}$ phosphate buffer and centrifuged at $16,000 \mathrm{xg}$ for $15 \mathrm{~min}$ at $4^{\circ} \mathrm{C}$. The supernatants were filtered through $0.22 \mu \mathrm{m}$ filters. Eighty microliters of supernatant was acidified by adding $40 \mu \mathrm{L}$ of $50 \%(\mathrm{v} / \mathrm{v})$ sulfuric acid. After vortexing and standing for $2 \mathrm{~min}$, the organic acids were extracted by adding $160 \mu \mathrm{L}$ of diethyl ether. Finally, the supernatant was collected for GC-MS analysis.

\section{Targeted bile acid quantitation}

UPLC-MS analysis of bile acids in the cecal contents of mice was performed on a Waters ACQUITY UPLC using a BEH HILIC column $(1.7 \mu \mathrm{m}, 2.1 \times 100 \mathrm{~mm})$ coupled with a Triple TOF 4000 system equipped with an ESI source operating in negative mode (AB SCIEX, USA). Data were analyzed with one-way ANOVA followed by a Tukey post hoc test. $10 \mathrm{mg}$ of cecal contents from mice was preweighed, mixed with $1 \mathrm{~mL}$ precooled methanol $\left(-20^{\circ} \mathrm{C}\right)$ and $100 \mathrm{mg}$ glass beads, and shaken for $1 \mathrm{~min}$ at $25 \mathrm{~Hz} / \mathrm{s}$, and this process was repeated at least two times. Next, samples were pulverized by ultrasonic wave for $30 \mathrm{~min}$ and then centrifuged at $12,000 \mathrm{rpm}$ for $10 \mathrm{~min}$ at $4^{\circ} \mathrm{C}$. Methanol $(580 \mu \mathrm{L})$ was added after collecting the supernatant $(20 \mu \mathrm{L})$ and vortexed for $30 \mathrm{~s}$. Finally, the supernatant was collected for LC-MS analysis.

\section{Targeted tryptophan metabolite quantitation}

UPLC-MS analysis of bile acids in the cecal contents of mice was performed on a Waters ACQUITY UPLC using an HSS T3 column $(1.8 \mu \mathrm{m}, 2.1 \times 100 \mathrm{~mm})$ coupled with a Q Exactive mass spectrometer equipped with an ESI source operating in negative mode (Thermo, USA). Data were analyzed with one-way ANOVA followed by a Tukey post hoc test. $30 \mathrm{mg}$ of fecal tissue from mice was preweighed and mixed with 500 $\mu \mathrm{L}$ of $50 \%$ acetonitrile, followed by thorough homogenization. Next, samples were kept on ice for $30 \mathrm{~min}$ and centrifuged at $12,000 \mathrm{rpm}$ for $10 \mathrm{~min}$ at $4^{\circ} \mathrm{C}$. The supernatant was collected, and the above steps were repeated three times. $200 \mu \mathrm{L}$ of $50 \%$ acetonitrile was added after collecting the supernatant and vacuum drying and centrifuged at 12,000 rpm for $10 \mathrm{~min}$. Finally, the supernatant was collected for LCMS analysis.

\section{Abbreviations}

KD: Ketogenic diet; SCFAs: Short-chain fatty acids; OGTT: Oral glucose tolerance test; H\&E: Hematoxylin and eosin; T2D: Type 2 diabetes; NAFLD: nonalcoholic fatty liver disease; LBP: Lipopolysaccharide (LPS)binding protein; RT-qPCR: reverse transcription qPCR; qPCR: Quantitative Real-time polymerase chain reaction; ASV: Amplicon sequence variant; QIIME: Quantitative insights into microbial ecology; PCoA: Principal coordinate analysis; PerMANOVA: Permutational multivariate analysis of variance; SPLS-DA: Sparse partial least-squares discriminant analysis; FDR; False discovery rate; LC-MS: Liquid chromatography-mass spectrometry; PCA: Principal component analysis; OPLS-DA: Orthogonal projection to latent structure-discriminant analysis; VIP: Variable importance in projection; BAs: Bile acids. 


\section{Declarations}

\section{Ethics approval and consent to participate}

The animal experimental procedures were approved by the Institutional Animal Care and Use Committee of Shanghai Jiao Tong University, Shanghai, China (No. 2018043 \& 2018106).

\section{Consent for publication}

Not applicable.

\section{Availability of data and materials}

The raw Illumine sequence data generated during the current study have been deposited to the NCBI under Bioproject accession \#PRJNA674951.

\section{Competing interests}

The authors declare that they have no competing interests.

\section{Funding}

This work was supported by the grants from the National Natural Science Foundation of China (31922003, 81871091) and the National Key Research and Development Project (2019YFA0905600).

\section{Authors' contributions}

$\mathrm{CHZ}$ conceived and designed the study. YL, XY, JZ, TYJ and ZYZ conducted the animal trial 1 and sample collection. YL, ZYW and MXG carried out the animal trial 2 and 3 and sample collection. YL conducted the physiological data analysis. YL, XY, ZYW and MXG prepared the DNA and conducted the sequencing of 16S rRNA gene. YL analyzed the sequencing data analysis and performed the metabolomics analysis. YL and $\mathrm{CHZ}$ wrote and revised the manuscript. All authors read and approved the final manuscript.

\section{Acknowledgements}

We thank Ting Xu, Yu Chen, Aolei Chen, Shuo Li and Yan Li for technical assistance.

\section{References}

1. Stafstrom CE, Rho JM. The ketogenic diet as a treatment paradigm for diverse neurological disorders. Front Pharmacol. 2012;3:59.

2. Paoli A, Rubini A, Volek JS, Grimaldi KA. Beyond weight loss: a review of the therapeutic uses of verylow-carbohydrate (ketogenic) diets. Eur J Clin Nutr. 2013;67(8):789-796. 
3. Dashti HM, Mathew TC, Hussein T, Asfar SK, Behbahani A, Khoursheed MA et al. Long-term effects of a ketogenic diet in obese patients. Exp Clin Cardiol. 2004;9(3):200-205.

4. Basciani S, Camajani E, Contini S, Persichetti A, Risi R, Bertoldi L et al. Very-Low-Calorie Ketogenic Diets with Whey, Vegetable or Animal Protein in Patients with Obesity: A Randomized Pilot Study. J Clin Endocrinol Metab. 2020.

5. Dashti HM, Mathew TC, Khadada M, Al-Mousawi M, Talib H, Asfar SK et al. Beneficial effects of ketogenic diet in obese diabetic subjects. Mol Cell Biochem. 2007;302(1-2):249-256.

6. Luukkonen PK, Dufour S, Lyu K, Zhang XM, Hakkarainen A, Lehtimäki TE et al. Effect of a ketogenic diet on hepatic steatosis and hepatic mitochondrial metabolism in nonalcoholic fatty liver disease. Proc Natl Acad Sci U S A. 2020;117(13):7347-7354.

7. Numao S, Kawano H, Endo N, Yamada Y, Konishi M, Takahashi M et al. Short-term low carbohydrate/high-fat diet intake increases postprandial plasma glucose and glucagon-like peptide-1 levels during an oral glucose tolerance test in healthy men. Eur J Clin Nutr. 2012;66(8):926-931.

8. Rosenbaum M, Hall KD, Guo J, Ravussin E, Mayer LS, Reitman ML et al. Glucose and Lipid Homeostasis and Inflammation in Humans Following an Isocaloric Ketogenic Diet. Obesity (Silver Spring). 2019;27(6):971-981.

9. Badman MK, Kennedy AR, Adams AC, Pissios P, Maratos-Flier E. A very low carbohydrate ketogenic diet improves glucose tolerance in ob/ob mice independently of weight loss. Am J Physiol Endocrinol Metab. 2009;297(5):E1197-1204.

10. Kennedy AR, Pissios P, Otu H, Roberson R, Xue B, Asakura K et al. A high-fat, ketogenic diet induces a unique metabolic state in mice. Am J Physiol Endocrinol Metab. 2007;292(6):E1724-1739.

11. Bielohuby M, Sisley S, Sandoval D, Herbach N, Zengin A, Fischereder M et al. Impaired glucose tolerance in rats fed low-carbohydrate, high-fat diets. Am J Physiol Endocrinol Metab. 2013;305(9):E1059-1070.

12. Garbow JR, Doherty JM, Schugar RC, Travers S, Weber ML, Wentz AE et al. Hepatic steatosis, inflammation, and ER stress in mice maintained long term on a very low-carbohydrate ketogenic diet. Am J Physiol Gastrointest Liver Physiol. 2011;300(6):G956-967.

13. Grandl G, Straub L, Rudigier C, Arnold M, Wueest S, Konrad D et al. Short-term feeding of a ketogenic diet induces more severe hepatic insulin resistance than an obesogenic high-fat diet. J Physiol. 2018;596(19):4597-4609.

14. Jornayvaz FR, Jurczak MJ, Lee HY, Birkenfeld AL, Frederick DW, Zhang D et al. A high-fat, ketogenic diet causes hepatic insulin resistance in mice, despite increasing energy expenditure and preventing weight gain. Am J Physiol Endocrinol Metab. 2010;299(5):E808-815.

15. Lamont BJ, Waters MF, Andrikopoulos S. A low-carbohydrate high-fat diet increases weight gain and does not improve glucose tolerance, insulin secretion or $\beta$-cell mass in NZO mice. Nutr Diabetes. 2016;6(2):e194.

16. Zhang X, Qin J, Zhao Y, Shi J, Lan R, Gan Y et al. Long-term ketogenic diet contributes to glycemic control but promotes lipid accumulation and hepatic steatosis in type 2 diabetic mice. Nutr Res. 
2016;36(4):349-358.

17. Ellenbroek JH, van Dijck L, Töns HA, Rabelink TJ, Carlotti F, Ballieux BE et al. Long-term ketogenic diet causes glucose intolerance and reduced $\beta$ - and $\alpha$-cell mass but no weight loss in mice. Am J Physiol Endocrinol Metab. 2014;306(5):E552-558.

18. Goldberg EL, Shchukina I, Asher JL, Sidorov S, Artyomov MN, Dixit VD. Ketogenesis activates metabolically protective $\gamma \delta T$ cells in visceral adipose tissue. Nature Metabolism. 2020;2(1):50-61.

19. Paoli A, Mancin L, Bianco A, Thomas E, Mota JF, Piccini F. Ketogenic Diet and Microbiota: Friends or Enemies? Genes (Basel). 2019;10(7).

20. Olson CA, Vuong HE, Yano JM, Liang QY, Nusbaum DJ, Hsiao EY. The Gut Microbiota Mediates the Anti-Seizure Effects of the Ketogenic Diet. Cell. 2018;173(7):1728-1741.e1713.

21. Ang QY, Alexander M, Newman JC, Tian Y, Cai J, Upadhyay V et al. Ketogenic Diets Alter the Gut Microbiome Resulting in Decreased Intestinal Th17 Cells. Cell. 2020;181(6):1263-1275.e1216.

22. Tilg $\mathrm{H}$, Zmora $\mathrm{N}$, Adolph TE, Elinav E. The intestinal microbiota fuelling metabolic inflammation. Nature Reviews Immunology. 2020;20(1):40-54.

23. Schugar RC, Huang X, Moll AR, Brunt EM, Crawford PA. Role of choline deficiency in the Fatty liver phenotype of mice fed a low protein, very low carbohydrate ketogenic diet. PLoS One. 2013;8(8):e74806.

24. Desai MS, Seekatz AM, Koropatkin NM, Kamada N, Hickey CA, Wolter M et al. A Dietary FiberDeprived Gut Microbiota Degrades the Colonic Mucus Barrier and Enhances Pathogen Susceptibility. Cell. 2016;167(5):1339-1353.e1321.

25. Nicolas GR, Chang PV. Deciphering the Chemical Lexicon of Host-Gut Microbiota Interactions. Trends Pharmacol Sci. 2019;40(6):430-445.

26. Moreira AP, Texeira TF, Ferreira AB, Peluzio Mdo C, Alfenas Rde C. Influence of a high-fat diet on gut microbiota, intestinal permeability and metabolic endotoxaemia. Br J Nutr. 2012;108(5):801-809.

27. Rafter JJ, Child P, Anderson AM, Alder R, Eng V, Bruce WR. Cellular toxicity of fecal water depends on diet. Am J Clin Nutr. 1987;45(3):559-563.

28. Degirolamo C, Modica S, Palasciano G, Moschetta A. Bile acids and colon cancer: Solving the puzzle with nuclear receptors. Trends Mol Med. 2011;17(10):564-572.

29. Belkaid Y, Hand TW. Role of the microbiota in immunity and inflammation. Cell. 2014;157(1):121141.

30. Sonnenburg JL, Bäckhed F. Diet-microbiota interactions as moderators of human metabolism. Nature. 2016;535(7610):56-64.

31. Natividad JM, Agus A, Planchais J, Lamas B, Jarry AC, Martin R et al. Impaired Aryl Hydrocarbon Receptor Ligand Production by the Gut Microbiota Is a Key Factor in Metabolic Syndrome. Cell Metab. 2018;28(5):737-749.e734.

32. Dutton SB, Sawyer NT, Kalume F, Jumbo-Lucioni P, Borges K, Catterall WA et al. Protective effect of the ketogenic diet in Scn1 a mutant mice. Epilepsia. 2011;52(11):2050-2056. 
33. Dorfman SE, Laurent D, Gounarides JS, Li X, Mullarkey TL, Rocheford EC et al. Metabolic implications of dietary trans-fatty acids. Obesity (Silver Spring). 2009;17(6):1200-1207.

34. Salmerón J, Hu FB, Manson JE, Stampfer MJ, Colditz GA, Rimm EB et al. Dietary fat intake and risk of type 2 diabetes in women. Am J Clin Nutr. 2001;73(6):1019-1026.

35. Kleiner DE, Brunt EM, Van Natta M, Behling C, Contos MJ, Cummings OW et al. Design and validation of a histological scoring system for nonalcoholic fatty liver disease. Hepatology. 2005;41(6):13131321.

36. Zhang L, Xue X, Zhai R, Yang X, Li H, Zhao L et al. Timing of Calorie Restriction in Mice Impacts Host Metabolic Phenotype with Correlative Changes in Gut Microbiota. mSystems. 2019;4(6).

37. Sorini C, Cosorich I, Lo Conte M, De Giorgi L, Facciotti F, Luciano R et al. Loss of gut barrier integrity triggers activation of islet-reactive $T$ cells and autoimmune diabetes. Proc Natl Acad Sci U S A. 2019;116(30):15140-15149.

38. Nadkarni MA, Martin FE, Jacques NA, Hunter N. Determination of bacterial load by real-time PCR using a broad-range (universal) probe and primers set. Microbiology. 2002;148(Pt 1):257-266.

39. Godon JJ, Zumstein E, Dabert P, Habouzit F, Moletta R. Molecular microbial diversity of an anaerobic digestor as determined by small-subunit rDNA sequence analysis. Appl Environ Microbiol. 1997;63(7):2802-2813.

40. Zhang Q, Wu Y, Wang J, Wu G, Long W, Xue Z et al. Accelerated dysbiosis of gut microbiota during aggravation of DSS-induced colitis by a butyrate-producing bacterium. Sci Rep. 2016;6:27572.

41. Bolyen E, Rideout JR, Dillon MR, Bokulich NA, Abnet CC, Al-Ghalith GA et al. Reproducible, interactive, scalable and extensible microbiome data science using QIIME 2. Nat Biotechnol. 2019;37(8):852857.

42. Callahan BJ, McMurdie PJ, Rosen MJ, Han AW, Johnson AJ, Holmes SP. DADA2: High-resolution sample inference from Illumina amplicon data. Nat Methods. 2016;13(7):581-583.

43. Callahan BJ, McMurdie PJ, Holmes SP. Exact sequence variants should replace operational taxonomic units in marker-gene data analysis. Isme j. 2017;11(12):2639-2643.

44. Pruesse E, Quast C, Knittel K, Fuchs BM, Ludwig W, Peplies J et al. SILVA: a comprehensive online resource for quality checked and aligned ribosomal RNA sequence data compatible with ARB. Nucleic Acids Res. 2007;35(21):7188-7196.

45. KA LC, Boitard S, Besse P. Sparse PLS discriminant analysis: biologically relevant feature selection and graphical displays for multiclass problems. BMC Bioinformatics. 2011;12:253.

46. Rohart F, Gautier B, Singh A, Le Cao KA. mixOmics: An R package for 'omics feature selection and multiple data integration. PLoS Comput Biol. 2017;13(11):e1005752.

47. Benjamini Y, Hochberg Y. Controlling the False Discovery Rate: A Practical and Powerful Approach to Multiple Testing. J R Statist Soc B. 1995;57:289-300.

48. Huang Y, Shen L, Jiang J, Xu Q, Luo Z, Luo Q et al. Metabolomic Profiles of Bovine Mammary Epithelial Cells Stimulated by Lipopolysaccharide. Sci Rep. 2019;9(1):19131. 
49. Wang $X$, Yang S, Li S, Zhao L, Hao Y, Qin J et al. Aberrant gut microbiota alters host metabolome and impacts renal failure in humans and rodents. Gut. 2020.

\section{Figures}
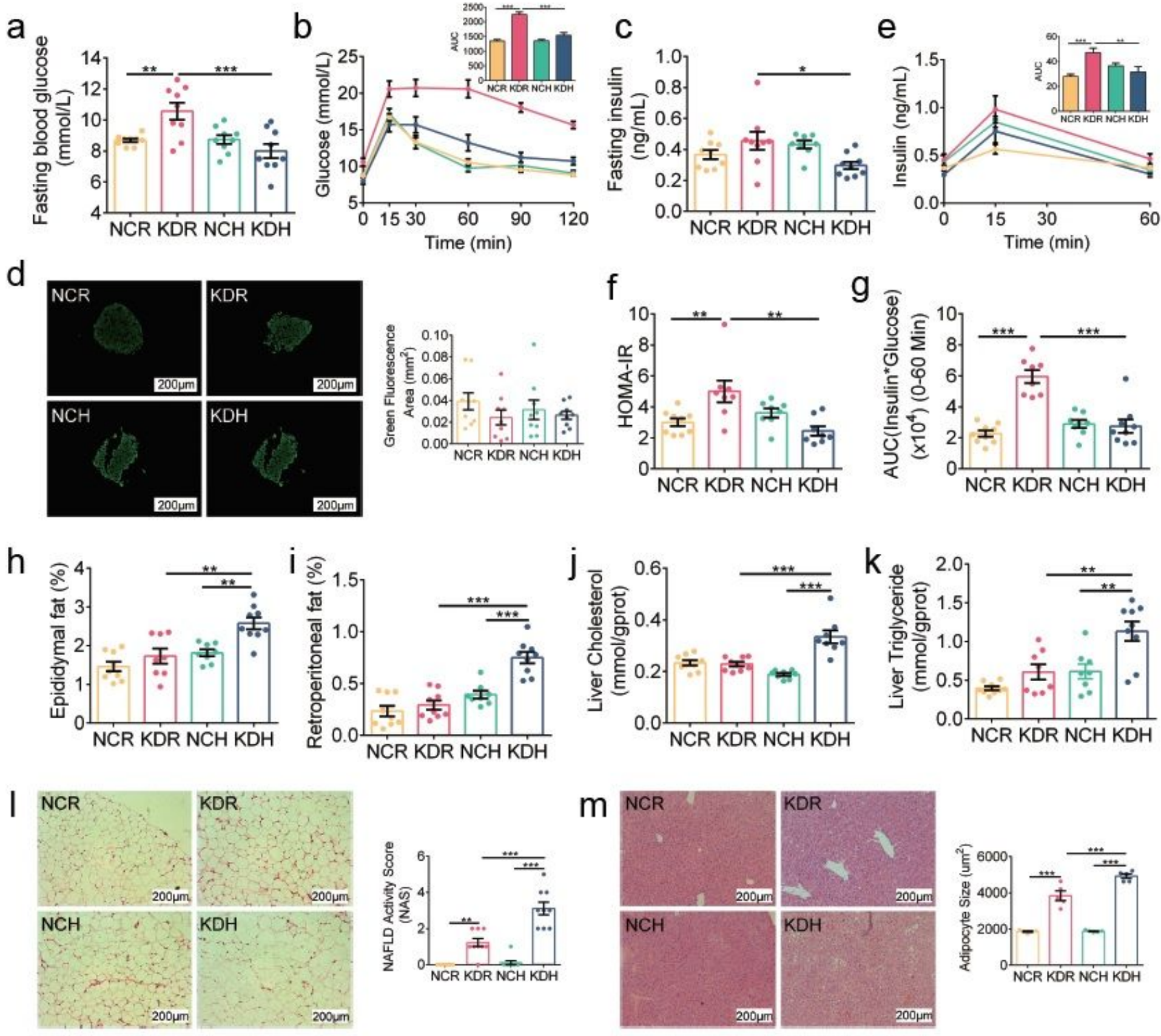

\section{Figure 1}

$\mathrm{KD}$ induced glucose intolerance and lipid accumulation in mice. a Fasting blood glucose. b Curves of blood glucose levels during oral glucose tolerance test (OGTT) and areas under the curve (AUC). c Fasting serum insulin. d Representative insulin immunofluorescence-stained (green) histological sections of pancreas (Scale bar $=200 \mu \mathrm{m}$ ) and calculated mean area of islet. e Serum insulin levels during OGTT 
and AUC. $\mathrm{f}$ Homeostasis model assessment of insulin resistance (HOMA-IR). $g$ Insulin resistance index (the product of the 0-60 min AUC of blood glucose and that of serum insulin in OGTT). h-i Epididymal and retroperitoneal fat mass (\% body weight). $j-k$ Concentrations of total cholesterol and triglyceride in liver. I Representative H\&E-stained histological sections of epididymal adipose tissue (eAT) (Scale bar $=200$ $\mu \mathrm{m})$ and calculated mean cell area of adipocytes ( $n=5$ per group). m Representative H\&E-stained histological sections of liver (Scale bar $=200 \mu \mathrm{m}$ ) and calculated histologic score (NAFLD Activity Score). Data are presented as the mean \pm SEM and analyzed using one-way ANOVA, followed by a Tukey post hoc test. ${ }^{*} P<0.05,{ }^{\star *} P<0.01,{ }^{\star \star *} P<0.001 . n=8-9$ for both groups for all analyses. 

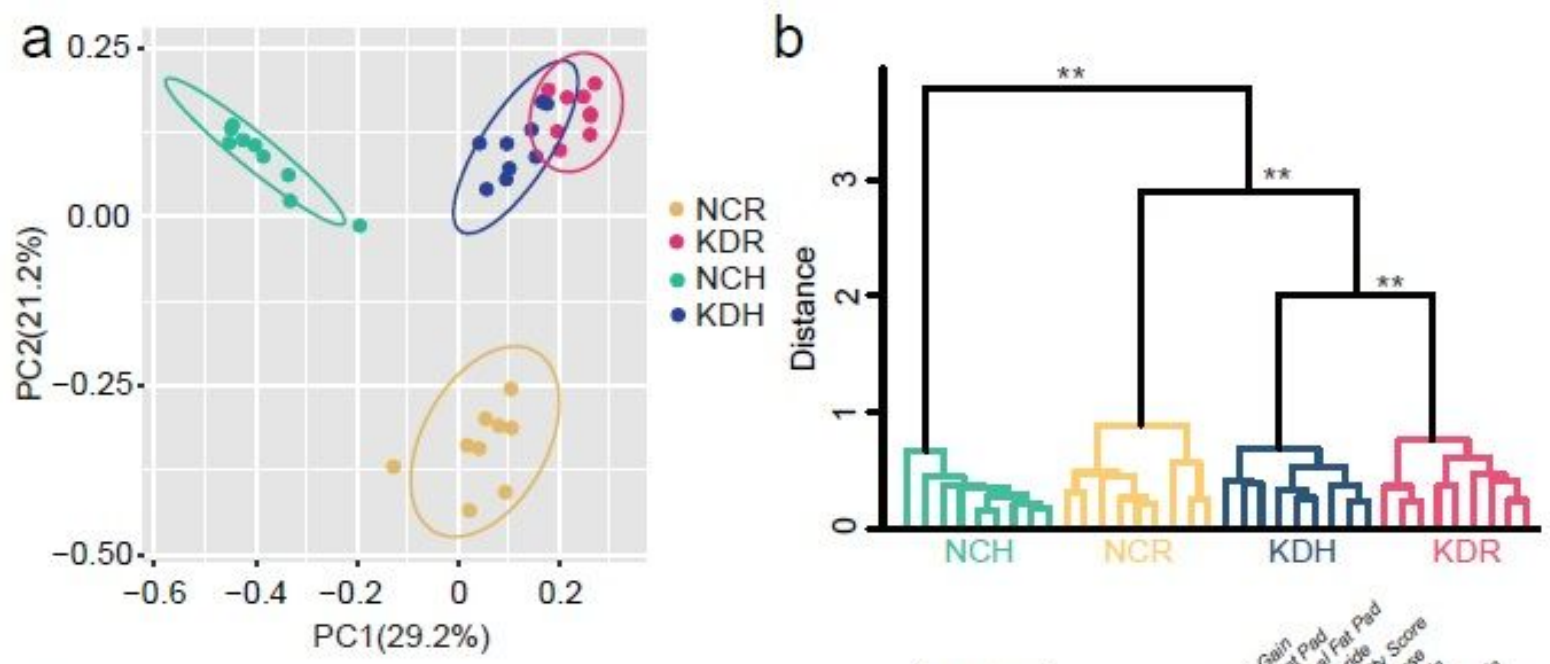

C
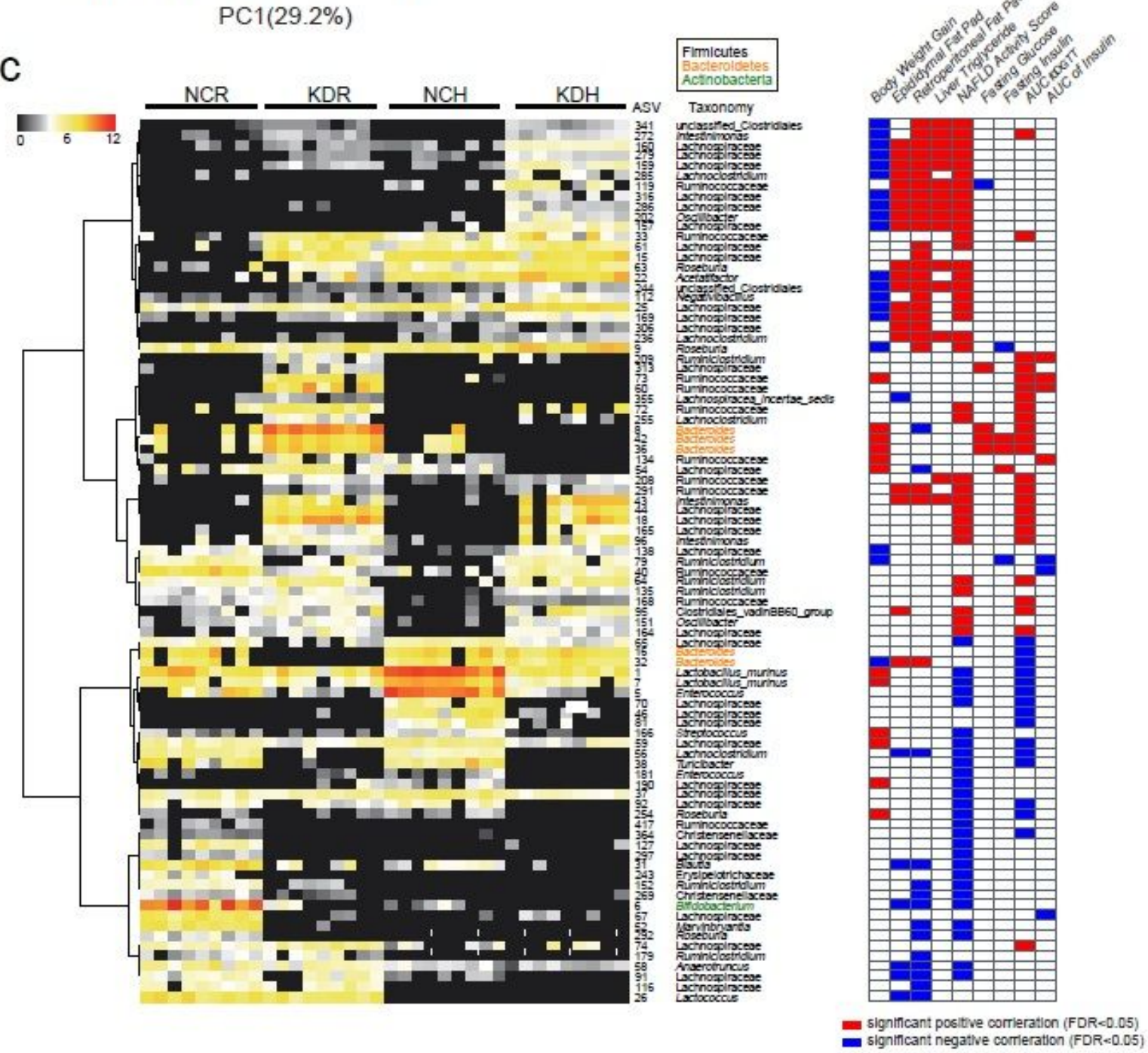

\section{Figure 2}

Two kinds of KD molded disparate gut microbiota in mice. a Principal-coordinate analysis (PCoA) plot of gut microbiota based on the Bray-Curtis distance. $b$ Clustering of the gut microbiota between different groups calculated with permutational multivariate analysis of variances (PERMANOVA; 999 permutations) test using Bray-Curtis distances. ${ }^{*} \mathrm{P}<0.01$. c Left, the heatmap represents the normalized and log2-transformed relative abundance of the 87 ASVs in each sample. These ASVs were clustered by 
the ward.D method. Right, Spearman correlations between the relative abundance of 87 ASVs and the host parameters related to glucose and lipid metabolism. $P$ values of correlations were adjusted by false discovery rate (FDR). Only when FDR $<0.05$, colors red and blue denote significant positive and negative correlation, respectively.

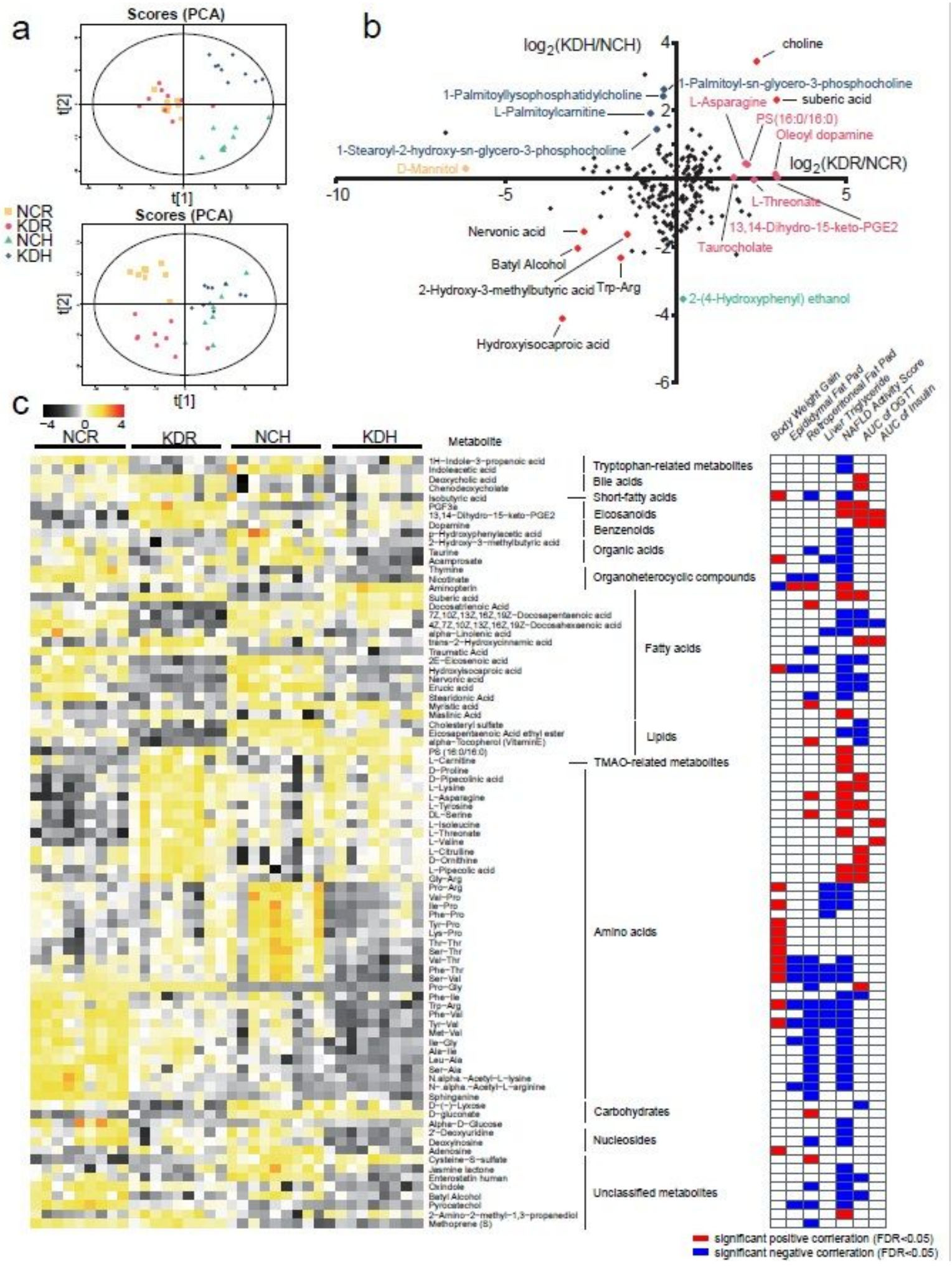

Figure 3 
Changes of the metabolome in mice fed with two kinds of KD. a Principal component analysis (PCA) plots of metabolic profiles. Top (ESI+), bottom (ESI-). b The fold ratio (log2-transformed) of the abundance of identified 226 metabolites in KDR to NCR mice, and $\mathrm{KDH}$ to NCH mice. The red spots represent the metabolites that enriched or decreased both in KDR and KDH group; and the rose red, orange, blue and mint green spots represent the metabolites that enriched in KDR, NCR, KDH and NCH mice, respectively. $c$ Left, the heatmap represents the abundance of the 85 metabolites in each sample. Right, Spearman correlations between the abundance of 85 metabolites and the host parameters related to glucose and lipid metabolism. $P$ values of correlations were adjusted by false discovery rate (FDR). Only when FDR $<0.05$, colors red and blue denote significant positive and negative association, respectively.

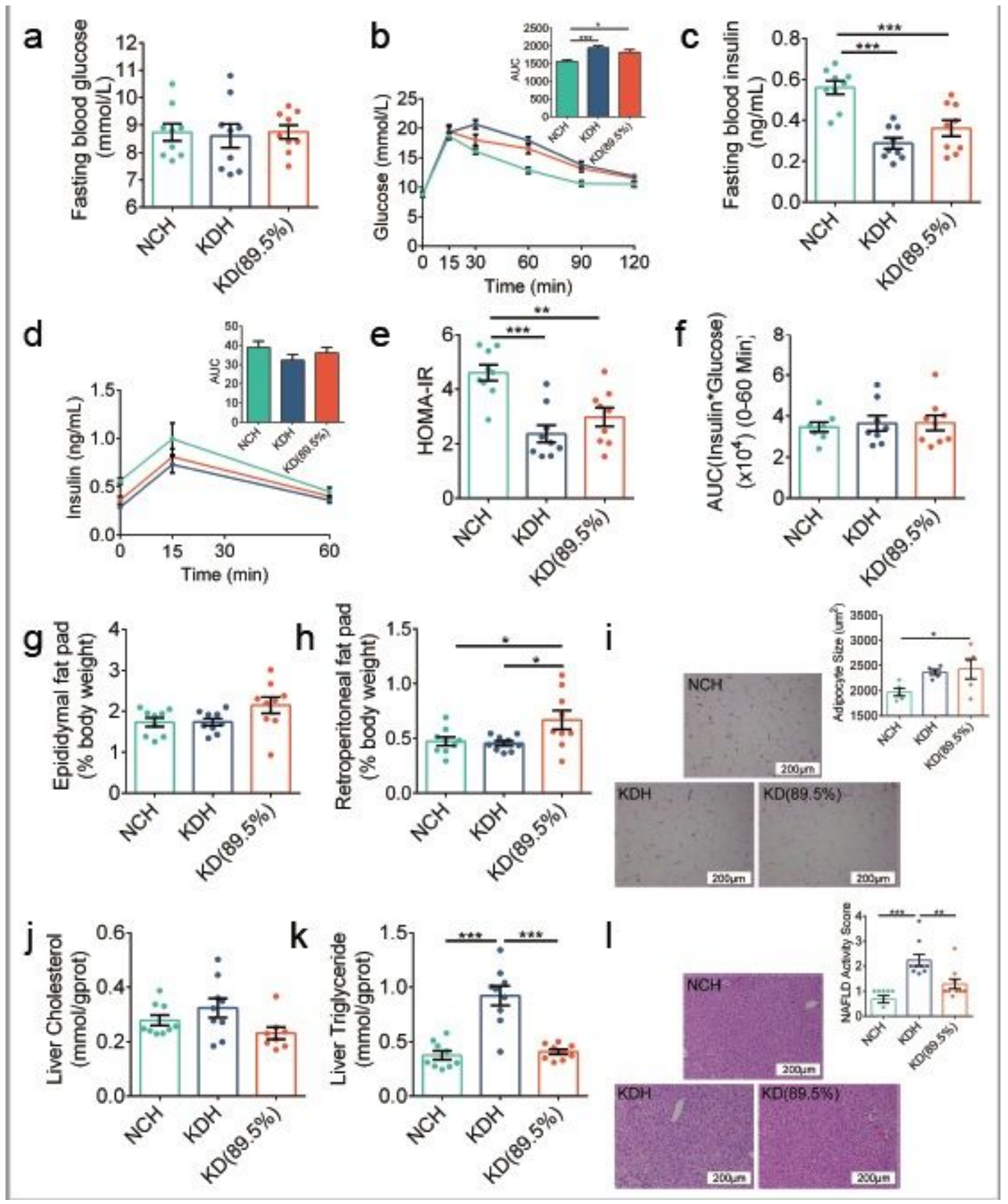

Figure 4 
The sources and proportions of fat in KDs affected the metabolic phenotypes in mice. a Fasting blood glucose. b Curves of blood glucose levels during OGTT and AUC. c Fasting serum insulin. $d$ Serum insulin levels during OGTT and AUC. e HOMA-IR. $\mathrm{f}$ Insulin resistance index. g-h Epididymal and retroperitoneal fat mass (\% body weight). i Representative H\&E-stained histological sections of eAT (Scale bar $=200 \mu \mathrm{m}$ ) and calculated mean cell area of adipocytes ( $n=5$ per group). $j-k$ Concentrations of total cholesterol and triglyceride in liver. I Representative H\&E-stained histological sections of liver (Scale bar $=200 \mu \mathrm{m}$ ) and NAFLD Activity Score. Data are presented as the mean \pm SEM and analyzed using one-way ANOVA, followed by a Tukey post hoc test. ${ }^{*} P<0.05,{ }^{*} P<0.01$, ${ }^{\star \star *} P<0.001 . n=8-9$ for both groups for all analyses. 


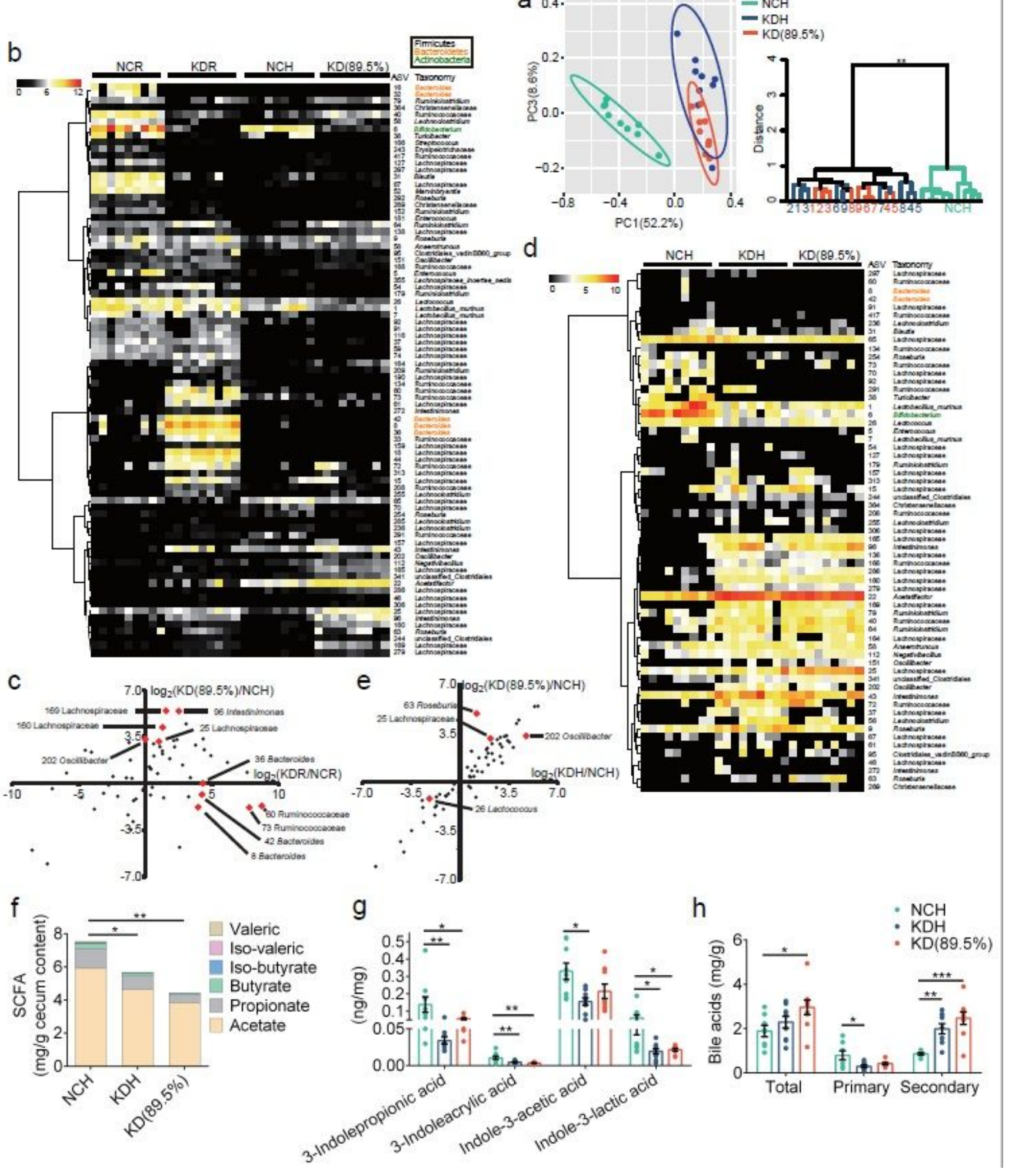

Figure 5

The gut microbiota and metabolites affected by the sources and proportions of fat in KDs. a Left, PCoA plot of gut microbiota based on the Bray-Curtis distance. Right, clustering of the gut microbiota between different groups calculated with PERMANOVA (999 permutations) test using Bray-Curtis distances. ${ }^{*} \mathrm{P}<$ 0.01. $b$ The heatmap represents the normalized and log2-transformed relative abundance of the 83 ASVs (the abundance of 4 ASVs was zero) in mice. The mice of these two KD groups were fed KDs with same 
proportion but different sources of fat. The ASVs were clustered by the ward.D method. c The fold ratio (log2-transformed) of the relative abundance of 83 ASVs in KD(89.5\%) to NCH mice, and KDR to NCR mice. The red spots represent the ASVs significantly different between two KD groups fed KDs with same proportion but different sources of fat. $\mathrm{d}$ The heatmap represents the normalized and log2-transformed relative abundance of the 63 ASVs (the abundance of 24 ASVs was zero) in mice. The mice of these two KD groups were fed KDs with same source but different proportions of fat. The ASVs were clustered by the ward.D method. e The fold ratio (log2-transformed) of the relative abundance of 63 ASVs in $\mathrm{KD}(89.5 \%)$ to $\mathrm{NCH}$ mice, and $\mathrm{KDH}$ to $\mathrm{NCH}$ mice. The red spots represent the ASVs significantly different between two KD groups fed KDs with same source but different proportions of fat. $f$ The concentrations of SCFAs in cecum content of mice ( $n=5-6$ per group). $g$ The concentrations of tryptophan metabolites in fecal of mice. $h$ The concentrations of bile acids (BAs) in cecum content of mice. For $f$-h, data are presented as the mean \pm SEM and analyzed using one-way ANOVA followed by a Tukey post hoc test. *P $<0.05, * * P<0.01, * \star * P<0.001 . n=8-9$ for both groups for all analyses. 

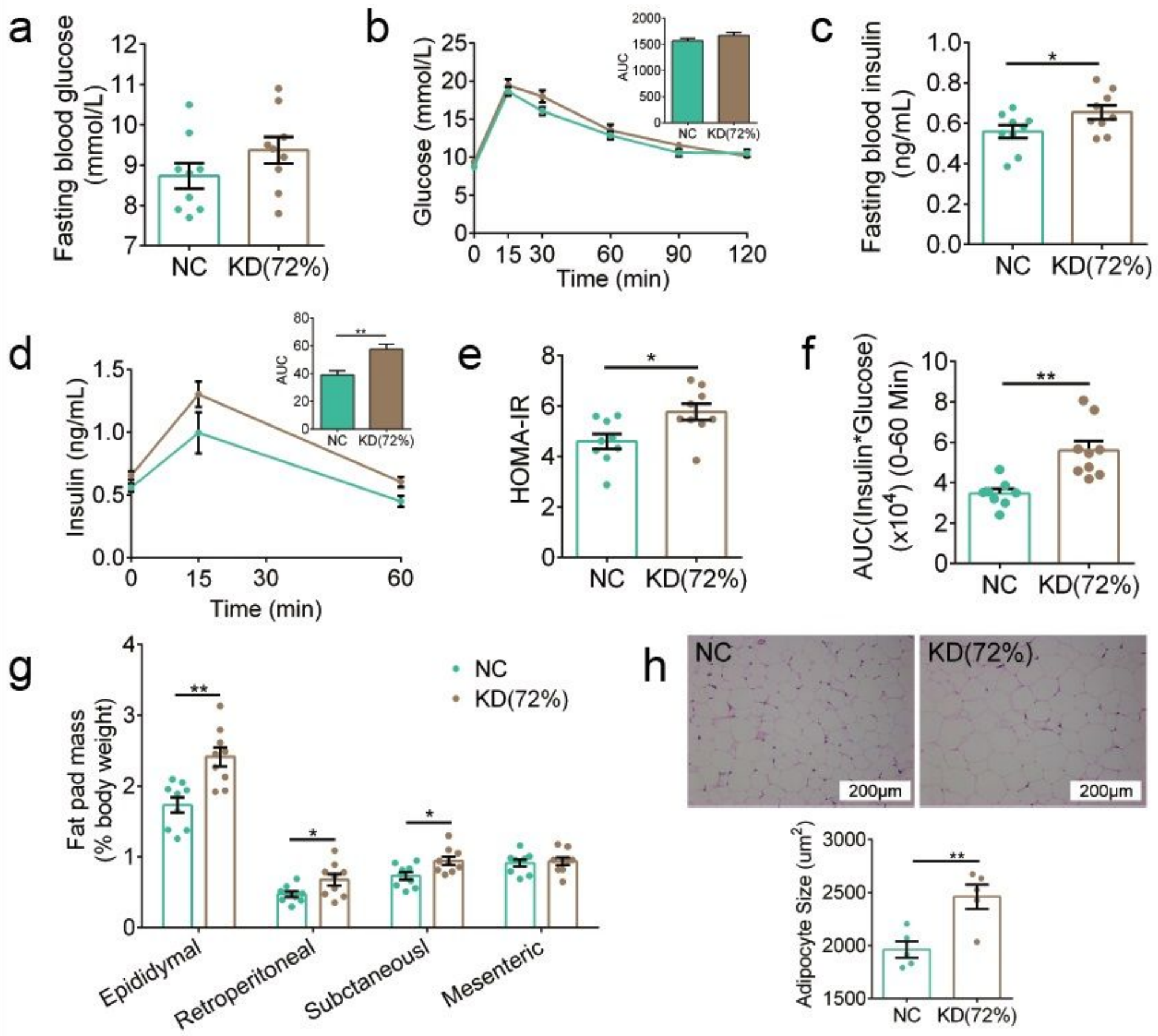

\section{Figure 6}

$\mathrm{KD}(72 \%)$ impaired metabolic health in mice. a Fasting blood glucose. b Curves of blood glucose levels during OGTT and AUC. c Fasting serum insulin. d Serum insulin levels during OGTT and AUC. e HOMA-IR. $\mathrm{f}$ Insulin resistance index. $\mathrm{g}$ Fat pad mass (\% body weight). h Representative H\&E-stained histological sections of eAT (Scale bar $=200 \mu \mathrm{m}$ ) and calculated mean cell area of adipocytes ( $n=5$ per group). Data are presented as the mean \pm SEM and analyzed using $T$ test. ${ }^{*} \mathrm{P}<0.01$. $n=8-9$ for both groups for all analyses. 


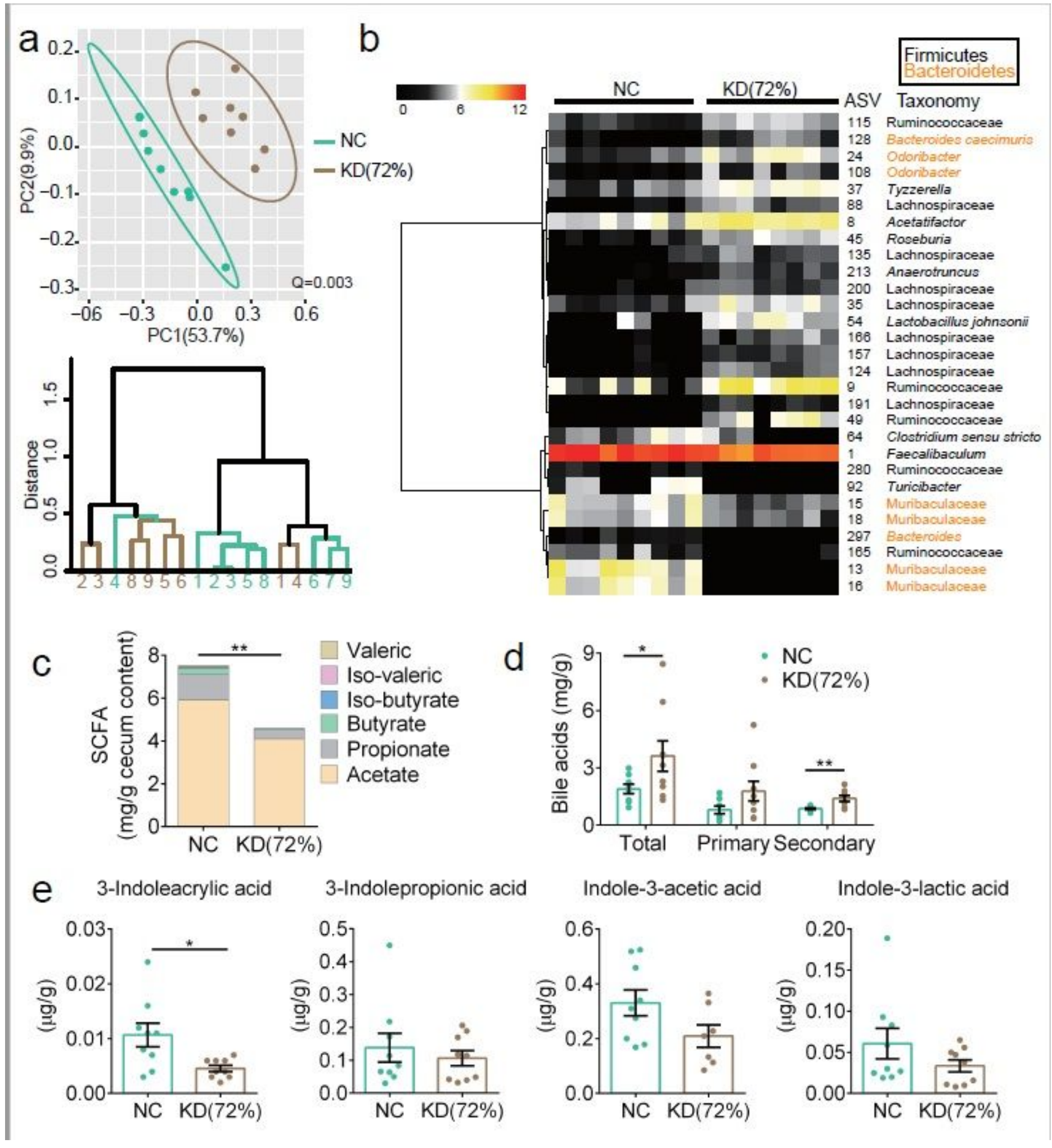

Figure 7

$\mathrm{KD}(72 \%)$ altered the gut microbiota and metabolites in mice. a Top, PCoA plot of gut microbiota based on the Bray-Curtis distance. Bottom, clustering of the gut microbiota between different groups calculated with PERMANOVA (999 permutations) test using Bray-Curtis distances. b The heatmap represents the normalized and log2-transformed relative abundance of the 29 ASVs significantly different between two groups. These ASVs were clustered by the ward.D method. c The concentrations of SCFAs in cecum 
content of mice ( $n=5-6$ per group). $d$ The concentrations of BAs in cecum content of mice. e The concentrations of tryptophan catabolites in fecal of mice. Data are presented as the mean \pm SEM and analyzed using $T$ test. ${ }^{*} \mathrm{P}<0.05,{ }^{*} \mathrm{P}<0.01 . \mathrm{n}=8-9$ for both groups for all analyses.

\section{Supplementary Files}

This is a list of supplementary files associated with this preprint. Click to download.

- Additionalfile.zip 